CLINICAL STUDY

\title{
Impaired subjective health status in chronic adrenal insufficiency: impact of different glucocorticoid replacement regimens
}

\author{
Benjamin Bleicken*, Stefanie Hahner ${ }^{1, *}$, Melanie Loeffler ${ }^{1}$, Manfred Ventz, Bruno Allolio ${ }^{1}$ and Marcus Quinkler \\ Clinical Endocrinology, Charité Campus Mitte, Charité University Medicine Berlin, Charitéplatz 1, D 10117 Berlin, Germany and ${ }^{1}$ Endocrinology and \\ Diabetes Unit, Department of Medicine I, University of Wuerzburg, 97080 Wuerzburg, Germany \\ (Correspondence should be addressed to M Quinkler; Email: marcus.quinkler@charite.de)
}

*(B Bleicken and S Hahner contributed equally to this work)

\begin{abstract}
Context: Recent studies have suggested that current glucocorticoid replacement therapies fail to fully restore well-being in patients with adrenal insufficiency (AI).

Objective: To investigate the effect of different glucocorticoid preparations used for replacement therapy on subjective health status (SHS) in AI.

Design and patients: In a cross-sectional study, primary and secondary AI patients were contacted by mail. Individual glucocorticoid replacement regimens, underlying diagnoses and comorbidities were verified by questionnaires and review of medical records. Patients were asked to complete three validated self-assessment questionnaires (Short Form 36 (SF-36), Giessen Complaint List (GBB-24), and Hospital Anxiety and Depression Scale). Results were compared with sex- and age-matched controls drawn from the questionnaire-specific reference cohort.

Results: Of the 883 patients identified, 526 agreed to participate in the study. Completed questionnaire sets were available from 427 patients (primary AI $n=232$; secondary AI $n=195$ ). AI patients showed significantly impaired SHS compared with controls irrespective of the glucocorticoid used for replacement. The only difference in SHS between patients on prednisolone (PR) and hydrocortisone (all patients and sub-analysis for primary AI) was significant higher bodily pain (lower Z-score in SF-36) in patients on PR $(P<0.05, P<0.01$ respectively). In patients with secondary AI, the PR group showed significantly $(P<0.05)$ less heart complaints (lower Z-score) in the GBB questionnaire compared with the cortisone acetate group.

Conclusions: Glucocorticoid replacement therapy with PR seems to be equivalent to hydrocortisone regarding SHS in patients with AI. However, SHS remains impaired in all patient groups suggesting a need for further improved glucocorticoid replacement strategies.
\end{abstract}

European Journal of Endocrinology 159 811-817

\section{Introduction}

Patients with chronic primary (PAI) and secondary adrenal insufficiency (SAI) rely on a lifelong, daily medical treatment with glucocorticoids, and in primary AI also with mineralocorticoids (1). Adding DHEA to the replacement regimen in patients with PAI and SAI is still in debate (2). Recently, it has been demonstrated that the patients with PAI and SAI show a significantly reduced subjective health status (SHS), irrespective of age, sex, concomitant disease, and primary or secondary origin of AI $(3,4)$. Importantly, also increased mortality has been described $(5,6)$. These observations have renewed the discussion of optimal replacement treatment in AI after about 50 years of almost unchanged regimens. The main focus of this discussion is glucocorticoid replacement as current replacement strategies lead to cortisol profiles differing profoundly from the physiological diurnal rhythm of serum cortisol.

In clinical practice, several different glucocorticoids are prescribed for hormone replacement therapy. Hydrocortisone (HC) is recommended by most authors as it represents the physiological glucocorticoid (7-9). However, even when given thrice daily, cortisol profiles only partially resemble the circadian profile due to very high peak levels followed by very low trough levels prior to the next oral dose. In PAI, this is also associated with intermittent excess of circulating ACTH. Cortisone acetate (CA), used in the study by Lovas et al. (3) in Norway, seems to have a lower serum cortisol peak and a delayed decline of cortisol levels $(10,11)$. However, cortisol generation may be occasionally affected by an impaired 11ß-hydroxysteroid reductase activity (12). 
Prednisolone (PR) is widely used for glucocorticoid replacement, usually given as a single morning dose of $5 \mathrm{mg}$. It has the advantage of a more sustained action compared with $\mathrm{HC}$ and a more convenient administration as a single morning dose. However, long-term side effects and the exact relative potency in humans remain still uncertain. The potency of PR is described to be four to five times higher than HC. Additionally, PR duration of action is $12-36 \mathrm{~h}$ compared with $6-10 \mathrm{~h}$ for HC. Limited data are available regarding long-term side effects of glucocorticoid replacement therapy (13). The currently available studies might suggest that bone loss is not influenced by the duration or type of steroid treatment $(14,15)$, but rather by the glucocorticoid dose used for chronic replacement (16). However, none of the studies had included large enough numbers to address this properly.

We have recently demonstrated reduced SHS in a large German cohort of patients with primary and secondary AI (4). Inadequate glucocorticoid replacement was suspected as a main cause of this impairment.

The aim of the present study was now to study potential differences in SHS and perceived influences on activities of daily life with regard to different glucocorticoid medications (PR, HC and CA) in this extended large German cohort of patients with PAI and SAI.

\section{Methods}

\section{Subjects}

Patients with AI from the Federal Republic of Germany and West Berlin with primary or SAI were usually treated with $\mathrm{HC}$, while patients from the former German Democratic Republic (GDR) received PR. This different medical substitution therapy in East- and West Germany has a predominately historical reason, due to former unavailability of $\mathrm{HC}$ in the GDR.

All AI patients are currently registered in the outpatient clinics of the Endocrine Departments of the Charité Campus Mitte Berlin and the University Hospital Wuerzburg or private endocrine practices in Berlin $(n=683)$, or are registered members of the German selfhelp network $(n=200)$. The patients were asked to participate in a postal survey. The study was approved by the ethical committees of the Charité Campus Mitte Berlin (permit no. ES1/037/06) and the University Hospital Wuerzburg (permit no. 45/04), and written informed consent was obtained from all patients prior to participation. Participating patients received four questionnaires, which they had to complete without consulting friends or family members, and were asked to return the completed questionnaires to the Endocrine Departments. Data regarding SHS from a subgroup of patients (132 patients with PAI and 78 with SAI) from Wuerzburg and the self-helping network have already been published (4).
The underlying diagnosis of AI was verified by review of the medical records. In addition, the following exclusion criteria were applied: AI due to long-term pharmacological glucocorticoid treatment, glucocorticoid doses above $7.5 \mathrm{mg}$ PR equivalent for reasons other than AI, adrenocortical carcinoma, congenital adrenal hyperplasia, adrenoleukodystrophy, and patients with less than 12 months duration of disease.

\section{Questionnaires}

Patients were asked to complete three different questionnaires and one self-established general registration form. Psychometric evaluation of patients was performed using three validated self-assessment SHS questionnaires: the social functioning (SF)-36, the brief form of the Giessen Complaint List (GBB-24), and the 'Hospital Anxiety and Depression Scale' (HADS). All three questionnaires are presented as a self-explanatory, multiple-choice self-assessment.

The SF-36 questionnaire is the most widely used generic instrument to assess health-related quality of life (17). It consists of eight multi-item domains representing physical functioning, role functioning physical, bodily pain (BP), general health perception, vitality, SF, role functioning emotional (RE), and mental health $(\mathrm{MH})$, as well as psychometrically based physical and $\mathrm{MH}$ summary measures. The domain scores range from 0 to 100 with higher values indicating better quality of life $(18,19)$. Reference data for SF-36 scores were obtained from the German National Health Survey (Bundesgesundheits Survey 1998, Robert Koch Institut Berlin 2000, Public use file BGS 98) comprising a representative random sample of 7124 subjects from the German population aged between 18 and 79 years (20). Higher -scores indicate less pain or less impaired functioning.

The short form of the GBB-24 questionnaire consists of 24 items defining four subscales (exhaustion tendency (et), gastric symptoms (gs), pain in the limbs (lp), and heart complaints (hc)) each including six items with ratings from 0 to 4 . Additionally, a global score of discomfort is calculated by adding up the four subscale scores. The maximum value for each subscale is 24 , and the global score 96. Higher scores indicate greater impairment of well-being (21).

The HADS is a 14-item, self-administered rating scale designed to measure anxiety and depression in physically ill individuals (22). Each item is scored as a number, with a maximum score of 21 for each subscale. Higher scores indicate higher levels of anxiety or depression. A cutoff value of 8 correlates with significant impairment and a cutoff value of 11 is indicative of major impairment, e.g. psychiatric disorders like major depression. Reference data for the GBB-24 $(n=2076)$ and HADS $(n=2081)$ were obtained from surveys performed by Braehler and co-workers $(21,23)$. 
Furthermore, a self-established general registration form collected data specifically matched for this survey. We were especially interested in issues concerning the patient's drug consumption (e.g. glucocorticoid use, daily dose), as well as the perceived influence of AI on activities of daily life. In addition, data were collected on duration and cause of AI, further medication, additional endocrine and general health problems, education, and occupational status.

Patients were grouped according to their sex, hormone replacement, as well as primary and secondary AI. Additionally, the duration of treatment and age of manifestation was taken into account. We compared the results of the three standardized questionnaires between the groups.

\section{Statistical analysis}

Comparison of quality of life scores between patients and matched controls was performed by MannWhitney U-test. Before comparison of the subgroups of patients with primary and secondary AI, which were inhomogeneous regarding age and sex distribution, adjustment for age and sex was performed by transformation of score values from patients and controls into age- (decade) and sex-adjusted Z-scores. Calculation of Z-scores was based on the complete data set from the respective normative groups. Differences in Z-scores, age, and body mass index (BMI) were subsequently analyzed by Mann-Whitney U-test.

Analyses were performed using the statistical software package SPSS, version 13.0 (SPSS Inc., Chicago, IL, USA). Significance was accepted if $P<0.05$.

\section{Results}

\section{Study cohort}

Of the 883 patients contacted, 526 patients $(60 \%)$ agreed to participate (Fig. 1) in the study. For further analysis of this study, 427 questionnaires were considered.

Data regarding SHS from a subgroup of patients (132 patients with PAI and 78 with SAI) from Wuerzburg and the self-helping network have already been published (4). However, in the published cohort only 18 patients received $C A$ and one patient $P R$. The subgroup of patients from Berlin and state Brandenburg included 100 new patients with PAI and 117 with SAI.

\section{Medical substitution}

We compared patients with PAI and SAI on treatment with HC, PR, or CA (Table 1).

Comparing all patients on $\mathrm{HC}$ versus patients on PR and CA regardless of sex and the cause of AI, the

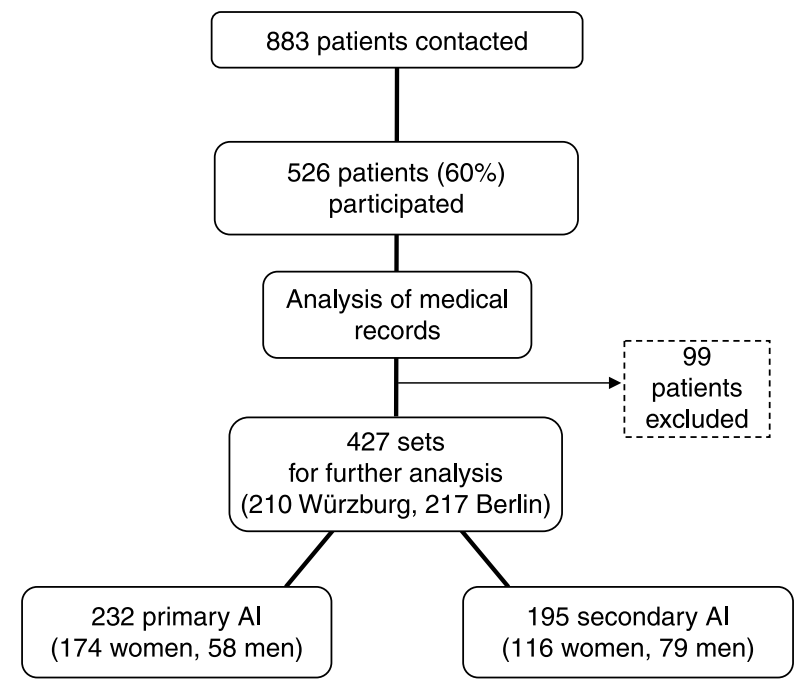

Figure 1 Flow sheet illustrating the recruitment for the study of 427 patients with adrenal insufficiency $(\mathrm{Al})$.

only significant $(P<0.05)$ difference was a higher BP (lower Z-score) in the SHS (SF-36) in patients on PR (Fig. 2a). The whole group of AI patients showed no considerable differences in anxiety or depression (HADS), or physical complaints (GBB-24; Figs 3a and 4a).

Sub-analysis of PAI patients showed that patients on PR had significantly higher BP (lower Z-score) than those on $\mathrm{HC}(P=0.006)$ and $\mathrm{CA}(P=0.008$; Fig. $2 \mathrm{~b})$. However, BP was not enhanced compared with controls. No differences were found in regard to anxiety or depression (HADS), or physical complaints (GBB-24) between different glucocorticoid therapy in patients with PAI (Figs 3b and 4b).

In patients with SAI, no difference was found between $\mathrm{HC}$ and PR replacement therapies in SHS (Figs 2c, 3c and $4 \mathrm{c})$. The only significant difference $(P<0.05)$ between $\mathrm{CA}$ and $\mathrm{PR}$ was found in the physical complaints questionnaire (GBB-24) with a lesser heart complaint (lower Z-score) in SAI patients on PR (Fig. 4c).

In a sex-specific sub-analysis, $\mathrm{PR}$ and CA were significantly better $(P<0.05)$ than $\mathrm{HC}$ with regard to the RE in the SHS (SF-36) in men (data not shown). In women on PR a higher BP $(P<0.05)$ in the SHS (SF-36) was found compared with women on $\mathrm{HC}$ (data not shown). Data analysis of the self-established general registration form specifically matched for this survey showed no significant difference between $\mathrm{HC}$ and PR (data not shown).

No significant differences in BMI were seen between patients on different glucocorticoid therapy (Table 1). Patients on PR were significantly older and had a longer duration of disease (Table 1). In PAI patients, no difference in the dosage of fludrocortisone therapy was found depending on the different glucocorticoid therapy (Fig. 5). 


\begin{tabular}{|c|c|c|c|c|c|c|c|c|c|c|c|c|}
\hline & \multicolumn{6}{|c|}{ PAI $(n=232)$} & \multicolumn{6}{|c|}{$\mathrm{SAI}(n=195)$} \\
\hline & \multicolumn{3}{|c|}{ Men $(n=58 ; 25 \%)$} & \multicolumn{3}{|c|}{ Women $(n=174 ; 75 \%)$} & \multicolumn{3}{|c|}{ Men $(n=79 ; 40 \%)$} & \multicolumn{3}{|c|}{ Women $(n=116 ; 60 \%)$} \\
\hline & $\mathrm{HC}$ & $\mathrm{CA}$ & PR & $\mathrm{HC}$ & $\mathrm{CA}$ & PR & $\mathrm{HC}$ & $\mathrm{CA}$ & PR & $\mathrm{HC}$ & $\mathrm{CA}$ & PR \\
\hline $\begin{array}{l}n \\
\text { Age (years) } \\
\text { Duration of } \\
\text { disease (years) }\end{array}$ & $\begin{array}{l}47 \\
47 \pm 2.4 \\
10 \pm 1.3\end{array}$ & $\begin{array}{l}7 \\
53 \pm 4.7 \\
25 \pm 6.4\end{array}$ & $\begin{array}{l}4 \\
65 \pm 10.7^{*} \\
20 \pm 4.6^{*}\end{array}$ & $\begin{array}{l}153 \\
48 \pm 1.1 \\
12 \pm 0.8\end{array}$ & $\begin{array}{l}5 \\
49 \pm 4.7 \\
18 \pm 6.4\end{array}$ & $\begin{array}{l}17 \\
60 \pm 2.5^{\dagger, \neq} \\
23 \pm 2.5^{\dagger}\end{array}$ & $\begin{array}{l}56 \\
54 \pm 2.2 \\
9 \pm 1.0\end{array}$ & $\begin{array}{l}2 \\
69 \pm 3.5 \\
26 \pm 15.0\end{array}$ & $\begin{array}{l}21 \\
61 \pm 2.7 \\
19 \pm 2.1^{\dagger}\end{array}$ & $\begin{array}{l}92 \\
51 \pm 1.5 \\
12 \pm 1.0\end{array}$ & $\begin{array}{l}5 \\
55 \pm 6.9 \\
15 \pm 7.6\end{array}$ & $\begin{array}{l}19 \\
62 \pm 2.5^{\star \star} \\
21 \pm 2.3^{\dagger}\end{array}$ \\
\hline $\begin{array}{l}\mathrm{BMI}\left(\mathrm{kg} / \mathrm{m}^{2}\right) \\
\text { On DHEA } \\
\text { treatment }(n=)\end{array}$ & $\begin{array}{l}25.6 \pm 0.6 \\
2\end{array}$ & $\begin{array}{l}26.4 \pm 1.9 \\
1\end{array}$ & $\begin{array}{l}25.9 \pm 2.3 \\
0\end{array}$ & $\begin{array}{l}23.7 \pm 0.3 \\
56\end{array}$ & $\begin{array}{l}22.1 \pm 1.0 \\
1\end{array}$ & $\begin{array}{l}25.0 \pm 0.9 \\
4\end{array}$ & $\begin{array}{l}26.8 \pm 0.8 \\
3\end{array}$ & $\begin{array}{l}24.4 \pm 2.6 \\
0\end{array}$ & $\begin{array}{l}27.0 \pm 0.7 \\
0\end{array}$ & $\begin{array}{l}26.4 \pm 0.6 \\
26\end{array}$ & $\begin{array}{l}25.7 \pm 1.8 \\
0\end{array}$ & $\begin{array}{l}28.3 \pm 1.4 \\
0\end{array}$ \\
\hline
\end{tabular}

Data are given as mean \pm S.E.M. ${ }^{*} P<0.05,{ }^{* *} P<0.005,{ }^{\dagger} P<0.001$ vs HC; $\neq=P<0.05$ vs CA. BMI, body mass index; DHEA, dehydroepiandrosterone.
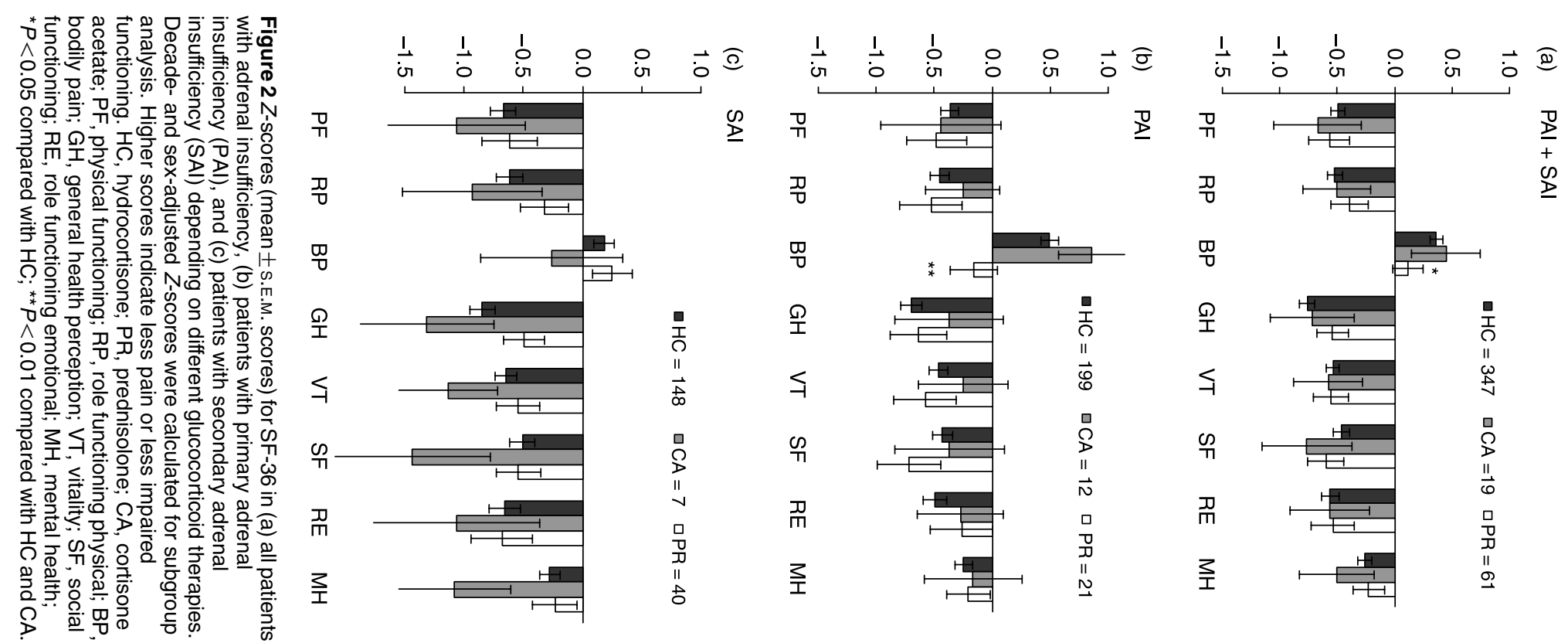
(a) $\mathrm{PAI}+\mathrm{SAI}$

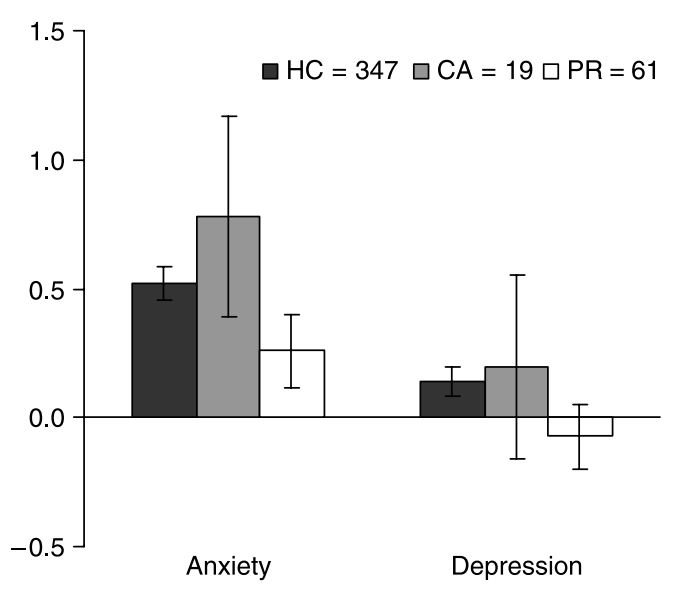

(b) PAI

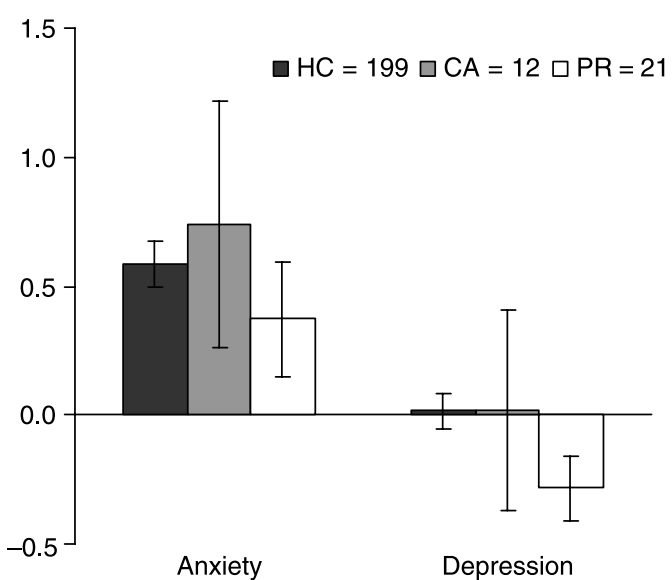

(c) SAI

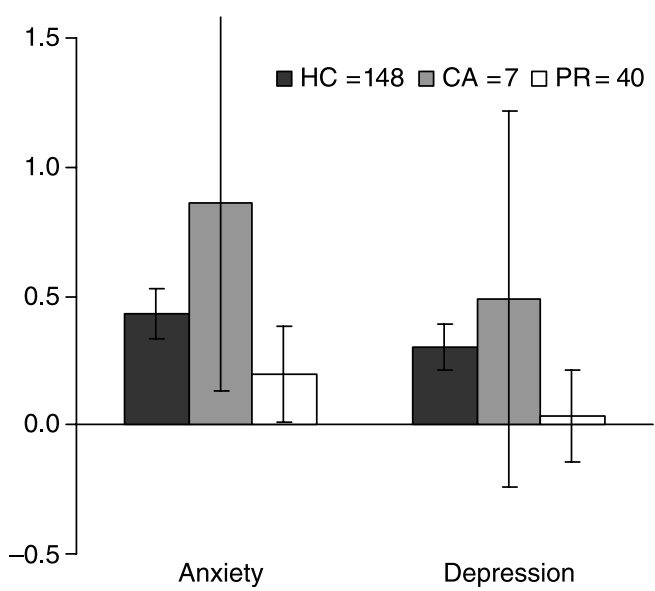

Figure 3 Z-scores (mean \pm s.E.M. scores) for HADS in (a) all patients with adrenal insufficiency, (b) patients with primary adrenal insufficiency (PAI), and (c) patients with secondary adrenal insufficiency (SAI) depending on different glucocorticoid therapies. Decade- and sex-adjusted Z-scores were calculated for subgroup analysis. Higher scores indicate higher levels of anxiety or depression. HC, hydrocortisone; PR, prednisolone; CA, cortisone acetate.

\section{Discussion}

Standard replacement therapy for chronic adrenal insufficiency (AI) consists of glucocorticoids (1), which clearly prevent life-threatening adrenal crisis. However, current replacement regimens fail to fully restore health-related quality of life in affected patients (3). An analysis of 989 patients with chronic AI from Denmark revealed a significantly higher rate of affective and depressive disorders compared with a control group of patients with osteoarthritis (24). Moreover, there is preliminary evidence from a large Swedish patient sample indicating that primary AI may even be associated with increased mortality (5), which had been demonstrated previously for patients with hypopituitarism including SAI (6). Recently, we have shown an impaired health-related SHS, irrespective of origin of disease or concomitant disease in 210 patients with primary and secondary AI (4). We hypothesized that non-physiological glucocorticoid replacement is the main cause of reduced health status in these patients.

The present study, which includes the already published subgroup of patients from Wuerzburg (4), confirms our recent findings of reduced SHS in this extended patient cohort of now 427 patients. Although $\mathrm{HC}, \mathrm{PR}$, and CA have different pharmacokinetic and also pharmacodynamic properties, SHS and daily performance was not influenced by these different replacement modes in a relevant manner.

We could detect no relevant differences in SHS between the glucocorticoid preparations used in our patients. A difference was seen in the overall cohort and in PAI patients with a higher pain perception in patients on PR compared with a decrease in those on CA or HC. However, pain perception in PR users did not differ from pain perception in the reference population. In our previous paper, we hypothesized that the reduced pain perception in patients with PAI might be due to the increased levels of POMC. Thus, a reduction of POMC levels by the longer acting PR might explain the observed differences between the $\mathrm{HC}$ and the PR group in the present study. However, no data on morning plasma ACTH concentrations were available to test this hypothesis.

As the mineralocorticoid effect of PR is less compared with $\mathrm{HC}$, higher doses of fludrocortisone were expected in the PR group. However, fludrocortisone doses were largely identical in the two subgroups suggesting different mineralocorticoid availability, which might also participate in the minor differences observed in this study.

The cohorts were slightly inhomogeneous regarding the age and disease duration with older subjects and longer disease duration in the PR group. Nevertheless, patients on PR presented with comparable SHS like patients on $\mathrm{HC}$ or CA. Furthermore, our analysis adjusted for age and sex by using Z-scores. We could not further detect a significant difference in BMI between the groups. BMI in the PR cohort was slightly higher but this might be attributed to the higher age. 

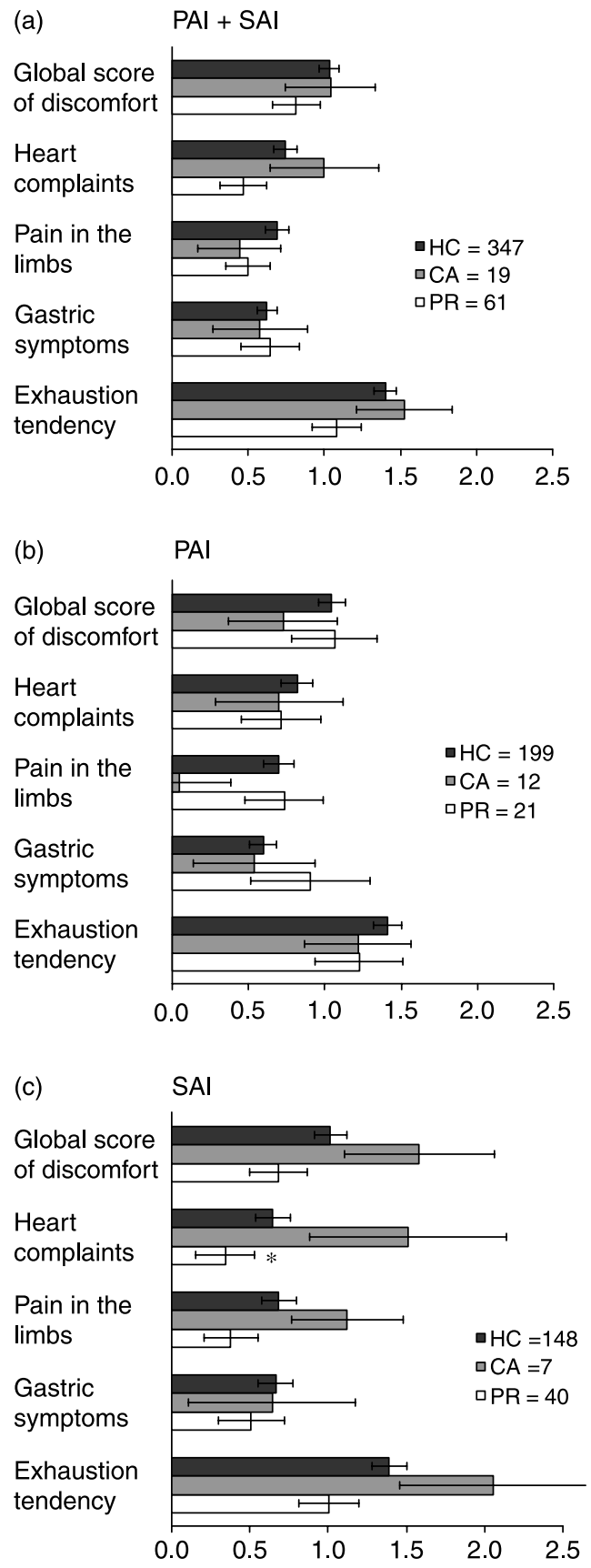

Figure 4 Z-scores (mean \pm s.E.M. scores) for GBB-24 in (a) all patients with adrenal insufficiency, (b) patients with primary adrenal insufficiency (PAI), and (c) patients with secondary adrenal insufficiency (SAI) depending on different glucocorticoid therapies. Decade- and sex-adjusted Z-scores were calculated for subgroup analysis. Higher scores indicate greater impairment of well-being. $\mathrm{HC}$, hydrocortisone; $\mathrm{PR}$, prednisolone, $\mathrm{CA}$, cortisone acetate; ${ }^{\star} P<0.05$ compared with $\mathrm{CA}$.

Altogether, in chronic glucocorticoid replacement therapy for AI, PR can be regarded as equivalent to $\mathrm{HC}$ concerning SHS. This is in contrast to the widely held opinion that long-acting glucocorticoids are inferior and should be avoided in AI. However, it is necessary to

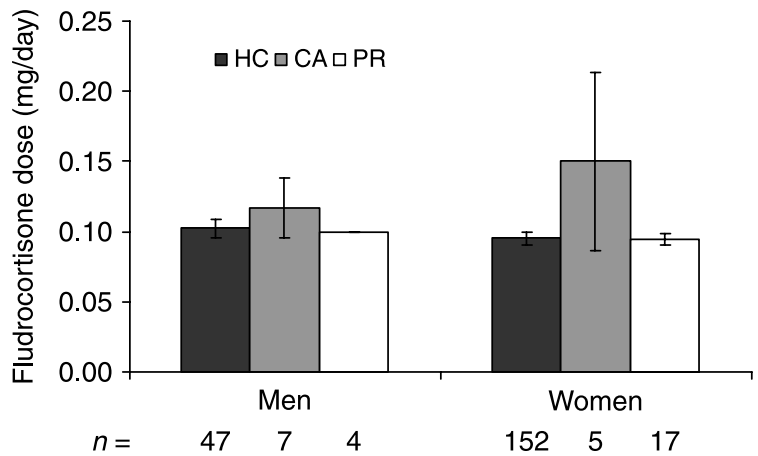

Figure 5 Daily fludrocortisone dose (mg/day) in patients with primary adrenal insufficiency depending on different glucocorticoid therapies ( $\mathrm{HC}$, hydrocortisone; CA, cortisone acetate; PR, prednisolone).

point out that this is a cross-sectional study, which shows only observational data. Possible limitations are that the cohort size might still be too small to detect real differences. In addition, we did not investigate long-term effects on other parameters such as bone turnover, metabolic parameters, or overall survival. However, in a small study there were no significant differences between bone mineral density in patients with $\mathrm{AI}$ treated with $\mathrm{HC}$ and those on PR (15). This indicates the need for prospective clinical trials.

The reason for the consistently impaired SHS in patients with AI remains to be elucidated. While in normal subjects cortisol is secreted in a pulsatile fashion with a clear diurnal rhythm $(25,26)$, this pattern is profoundly different in patients with AI receiving current replacement regimens (13). In particular, patients take their first $\mathrm{HC}$ dose in the morning starting with very low cortisol levels, whereas in normal subjects a steep cortisol rise is found in the hours before waking. Accordingly, patients with PAI typically exhibit increased plasma ACTH concentrations prior to the first morning dose. Thus, the most pronounced difference to normal subjects occurs in the hours between midnight and waking. New timed release glucocorticoid preparations may allow the treatment to better mimic the early morning cortisol rise prior to waking in the patients with $\mathrm{AI}$ and may hold the potential to positively affect the quality of life in AI (27). This view is supported by a recent short open-label pilot study in seven patients with Addison's disease. In this study, administration of $\mathrm{HC}$ via continuous s.c. infusion successfully re-established the physiological circadian variation and allowed reduction of the glucocorticoid dose. Treatment was well tolerated and led to an improvement of SHS (28). Furthermore, a timed release PR preparation was recently used in patients with rheumatoid arthritis showing that glucocorticoid release prior to waking significantly reduced disease activity compared with an identical morning dose of standard PR (29).

In conclusion, PR is equivalent to $\mathrm{HC}$ as glucocorticoid replacement therapy regarding SHS, which is significantly 
impaired in AI compared with sex- and age-matched controls. An optimal glucocorticoid replacement awaits the availability of a glucocorticoid formulation that can replicate the normal circadian rhythm and replace physiological cortisol production. Future interventional trials are necessary to explore the role of these more physiological glucocorticoid replacement strategies in AI.

\section{Declaration of interest}

There is no conflict of interest that could be perceived as prejudicing the impartiality of the research reported.

\section{Funding}

This research did not receive any specific grant from any funding agency in the public, commercial or not-for-profit sector.

\section{Acknowledgements}

We are indebted to Sven Diederich, Reinhard Finke, and Ullrich Bogner, Berlin, for recruitment of patients. We thank Prof. J Hensen, Prof. C Schöfl, Mrs Hummel, Mrs Jalowski, and Mrs Stahl from the German Self-Help Network for Pituitary and Adrenal Diseases 'Netzwerk Hypophysen- und Nebennierenerkrankungen e. V.' for their invaluable support by contacting the patients of this network and distributing the questionnaires. We thank Prof. E. Braehler, Dept of Psychotherapy and Psychosomatic Medicine, University Hospital Leipzig, Germany, for providing the normative data of the GBB-24 and HADS scores from the general German population.

\section{References}

1 Arlt W \& Allolio B. Adrenal insufficiency. Lancet 2003361 1881-1893.

2 Allolio B, Arlt W \& Hahner S. DHEA: why, when, and how much DHEA replacement in adrenal insufficiency. Annales d'Endocrinologie $200768268-273$.

3 Lovas K, Loge JH \& Husebye ES. Subjective health status in Norwegian patients with Addison's disease. Clinical Endocrinology 200256 581-588.

4 Hahner S, Loeffler M, Fassnacht M, Weismann D, Koschker AC, Quinkler M, Decker O, Arlt W \& Allolio B. Impaired subjective health status in 256 patients with adrenal insufficiency on standard therapy based on cross-sectional analysis. Journal of Clinical Endocrinology and Metabolism 200792 3912-3922.

5 Bergthorsdottir R, Leonsson-Zachrisson M, Oden A \& Johannsson G. Premature mortality in patients with Addison's disease: a population-based study. Journal of Clinical Endocrinology and Metabolism 200691 4849-4853.

6 Tomlinson JW, Holden N, Hills RK, Wheatley K, Clayton RN, Bates AS, Sheppard MC \& Stewart PM. Association between premature mortality and hypopituitarism. West Midlands Prospective Hypopituitary Study Group. Lancet 2001357 425-431.

7 Ten S, New M \& Maclaren N. Clinical review 130: Addison's disease. Journal of Clinical Endocrinology and Metabolism 200186 2909-2922.

8 Oelkers W. Adrenal insufficiency. New England Journal of Medicine 1996335 1206-1212.

9 Jeffcoate W. Assessment of corticosteroid replacement therapy in adults with adrenal insufficiency. Annals of Clinical Biochemistry 199936 151-157.

10 Feek CM, Ratcliffe JG, Seth J, Gray CE, Toft AD \& Irvine WJ. Patterns of plasma cortisol and ACTH concentrations in patients with Addison's disease treated with conventional corticosteroid replacement. Clinical Endocrinology 198114 451-458.
11 Nickelsen T, Schulz F \& Demisch K. Studies on cortisol substitution therapy in patients with adrenal insufficiency. Experimental and Clinical Endocrinology 198382 35-41.

12 Nordenström A, Marcus C, Axelson M, Wedell A \& Ritzén EM. Failure of cortisone acetate treatment in congenital adrenal hyperplasia because of defective 11 $\beta$-hydrosteroid dehydrogenase reductase activity. Journal of Clinical Endocrinology and Metabolism 199984 1210-1213.

13 Crown A \& Lightman S. Why is the management of glucocorticoid deficiency still controversial: a review of the literature. Clinical Endocrinology 200563 483-492.

14 Jodar E, Valdepenas MP, Martinez G, Jara A \& Hawkins F. Longterm follow-up of bone mineral density in Addison's disease. Clinical Endocrinology 200358 617-620.

15 Valero MA, Leon M, Ruiz Valdepenas MP, Larrodera L, Lopez MB, Papapietro K, Jara A \& Hawkins F. Bone density and turnover in Addison's disease: effect of glucocorticoid treatment. Bone and Mineral 199426 9-17.

16 Zelissen PM, Croughs RJ, van Rijk PP \& Raymakers JA. Effect of glucocorticoid replacement therapy on bone mineral density in patients with Addison disease. Annals of Internal Medicine 1994 120 207-210.

17 Ware JE Jr \& Sherbourne CD. The MOS 36-Item, Short-Form Health Survey (SF-36), I. Conceptual framework and item selection. Medical Care 199230 473-483.

18 Nemeth G. Health related quality of life outcome instruments. European Spine Journal 200615 (Suppl 1) S44-S51.

19 Garratt A, Schmidt L, Mackintosh A \& Fitzpatrick R. Quality of life measurement: bibliographic study of patient assessed health outcome measures. BMJ $2002 \mathbf{3 2 4} 1417$.

20 Ellert U \& Bellach BM. The SF-36 in the federal health surveydescription of a current normal sample. Gesundheitswesen 1999 61 S184-S190.

21 Brahler E, Schumacher J \& Brahler C. First All-Germany Standardization of the Brief Form of the Gissen Complaints Questionnaire GBB-24. Psychotherapie, Psychosomatik, Medizinische Psychologie 200050 14-21.

22 Zigmond AS \& Snaith RP. The hospital anxiety and depression scale. Acta Psychiatrica Scandinavica 198367 361-370.

23 Hinz A, Brahler E, Schwarz R, Schumacher J \& Stirn A. How useful is the calculation of total scores for Questionnaires concerning health related quality of life? Psychotherapie, Psychosomatik, Medizinische Psychologie 200555 221-228.

24 Thomsen AF, Kvist TK, Andersen PK \& Kessing LV. The risk of affective disorders in patients with adrenocortical insufficiency. Psychoneuroendocrinology 200631 614-622.

25 Chrousos GP. Ultradian, circadian, and stress-related hypothalamic-pituitary-adrenal axis activity - a dynamic digitalto-analog modulation. Endocrinology 1998139 437-440.

26 Krieger DT, Allen W, Rizzo F \& Krieger HP. Characterization of the normal temporal pattern of plasma corticosteroid levels. Journal of Clinical Endocrinology and Metabolism 197132 266-284.

27 Newell-Price J, Whiteman M, Rostami-Hodjegan A, Darzy K, Shalet S, Tucker GT \& Ross RJ. Modified-release hydrocortisone for circadian therapy: a Proof-of-Principle Study in Dexamethasone-Suppressed Normal Volunteers. Clinical Endocrinology 200868 130-135.

28 Lovas K \& Husebye ES. Continuous subcutaneous hydrocortisone infusion in Addison's disease. European Journal of Endocrinology 2007157 109-112.

29 Buttgereit F, Doering G, Schaeffler A, Witte S, Sierakowski S, Gromnica-Ihle E, Jeka S, Krueger K, Szechinski J \& Alten R. Efficacy of modified-release versus standard prednisone to reduce duration of morning stiffness of the joints in rheumatoid arthritis (CAPRA-1): a double-blind, randomised controlled trial. Lancet 2008371 205-214.

Received 16 September 2008

Accepted 17 September 2008 